\title{
Salivary diagnosis of measles: a study of notified cases in the United Kingdom, 1991-3
}

\author{
David W G Brown, Mary E B Ramsay, Alison F Richards, Elizabeth Miller
}

\author{
Abstract \\ Objectives-To validate a method for salivary \\ diagnosis of measles and to assess the diagnostic \\ accuracy of notified cases of measles. \\ Design-Blood and saliva samples were collected \\ within 90 days of onset of symptoms from patients \\ clinically diagnosed as having measles and tested for \\ specific IgM by antibody capture radioimmunoassay. \\ Setting-17 districts in England and one in \\ southern Ireland during August 1991 to February \\ 1993.
}

Subjects -236 children and adults with measles notified by a general practitioner.

Results-Specific IgM was detected in serum in only $85(36 \%)$ of the 236 cases. In cases associated with outbreaks and tested within six weeks of onset, $53 / 57(93 \%)$ of samples were IgM positive, thereby confirming the sensitivity of serum IgM detection as a marker of recent infection. The serological confirmation rate was lower in cases with a documented history of vaccination $(13 / 87 ; 15 \%)$ than in those without $(70 / 149 ; 47 \%)$ and varied with age, being lowest in patients under a year, of whom only 4/36 $(11 \%)$ were confirmed. Measles specific IgM was detected in $71 / 77(92 \%)$ of adequate saliva samples collected from patients with serum positive for IgM. In cases where measles was not confirmed, 6/101 had rubella specific IgM and $5 / 132$ had human parvovirus B19 specific IgM detected in serum.

Conclusions-The existing national surveillance system for measles, which relies on clinically diagnosed cases, lacks the precision required for effective disease control. Saliva is a valid alternative to serum for IgM detection, and salivary diagnosis could play a major role in achieving measles elimination. Rubella and parvovirus B19 seem to be responsible for a minority of incorrectly diagnosed cases of measles in the United Kingdom and other infectious causes of measles-like illness need to be sought.

Enteric and Respiratory

Virus Division, Virus

Reference Division,

PHLS Central Public

Health Laboratory,

London NW9 5HT

David W G Brown,

consultant virologist

Alison F Richards, medical

laboratory scientific officer

Immunisation Division, PHLS Communicable

Disease Surveillance

Centre, London NW9 5EQ

Mary E B Ramsay, research

registrar

Elizabeth Miller, consultant epidemiologist

Correspondence and requests for reprints to: Dr Miller.

$B M 7$ 1994;308:1015-7

\section{Introduction}

Measles vaccine was replaced by combined measles, mumps, and rubella vaccine in 1988 , with the aim of eliminating all three diseases from the United Kingdom. ${ }^{1}$ Since the introduction of the vaccine, coverage has improved steadily with $93 \%$ of children now being vaccinated by 2 years of age ${ }^{2}$; the annual number of notified measles cases has fallen lower than ever before. ${ }^{3}$ Despite the overall reduction in the incidence of measles, outbreaks in older children have recently been reported, suggesting that a two dose strategy may be needed to ensure elimination. ${ }^{4}$ In addition, the increase in the proportion of notified cases in which patients are aged under 1 year from $9 \%$ in 1987 to $28 \%$ in $1991^{3}$ may indicate a need for a reduction in the age at vaccination. As measles becomes less common, however, it is to be expected that an increasing proportion of notified cases will not be due to measles infection. Accurate monitoring of the progress towards elimination of the disease and identification of the important reservoirs of infection may therefore require extensive laboratory confirmation of cases. Existing laboratory methods rely largely on the detection of significant rises in measles antibody titre or the detection of measles specific IgM and are impractical for widespread use in children as blood samples are required. A non-invasive method of confirming measles infection would be of considerable clinical and epidemiological value.

We have developed an antibody capture method for detecting IgM specific to measles, mumps, and rubella viruses in the saliva of patients who have had the disease which, when used in a hospital based study, proved both sensitive and specific. ${ }^{5}$ This study was designed to validate the salivary assay under conditions of routine community use and to investigate the diagnostic accuracy of notified cases of measles.

\section{Methods}

Consultants in communicable disease control in 18 collaborating districts were provided with kits containing equipment for the collection of saliva and blood spot samples from patients recently notified as having measles. On receipt of a notification, the consultant in communicable disease control sent a kit to the reporting doctor, requesting samples from the patient, preferably within 60 days of onset of illness. Saliva samples were taken by the doctor or practice nurse at a home or clinic visit by wiping a specially designed sponge swab $^{6}$ around the gum margin for about a minute. At the same time, a few drops of blood from a heel or finger prick were collected on to a filter paper strip. Saliva and blood samples were posted with a simple request form to the Public Health Laboratory Service Virus Reference Division. Vaccination histories of patients were obtained from general practice and health authority records.

Saliva was extracted from the sponge swabs into $1 \mathrm{ml}$ of phosphated buffered saline, $\mathrm{pH} 7 \cdot 2$, containing $0.2 \%$ Tween 20 and $10 \%$ fetal calf serum by vortexing and centrifugation. Extracted saliva was stored at $-30^{\circ} \mathrm{C}$ before testing. Serum was extracted from a measured area of filter paper into $200 \mu l$ phosphate buffered saline by using established methods. ${ }^{7}$ Saliva and serum samples were tested for measles specific IgM by antibody capture radioimmunoassay based on semipurified tissue culture grown virus and a measles specific monoclonal antibody. ${ }^{5}$ Saliva or serum specimens were considered positive for $\operatorname{IgM}$ if the $T: N$ ratio exceeded 3.0.5 Saliva extracts were tested neat and serum samples at a final dilution of 1 in 64 . Saliva specimens were also tested for total IgG content by radial immunodiffusion (Nanorid-IgG-Ul, range $0.5-5 \mathrm{mg} / \mathrm{l}$, The Binding Site Ltd, Birmingham) to establish the adequacy of the sample. Selected serum samples were tested for rubella specific IgM $^{5}$ and human parvovirus $\mathrm{B} 19 \mathrm{IgM}^{8}$ by using previously described methods.

The data were analysed by using logistic regression with factors for age, sex, vaccination status, and case type (sporadic or associated with outbreak). Time between onset of symptoms and antibody testing was fitted both as a continuous variable and as a factor with 
TABLE I-Proportion of notified cases of measles confirmed by detection of specific IgM antibody in serum

\begin{tabular}{lcccccc}
\hline & \multicolumn{2}{c}{ Not vaccinated } & & \multicolumn{2}{c}{$\begin{array}{c}\text { Documented previous } \\
\text { vaccination }\end{array}$} \\
\cline { 2 - 3 } \cline { 6 - 7 } $\begin{array}{l}\text { Age } \\
\text { (years) }\end{array}$ & $\begin{array}{c}\text { No } \\
\text { tested }\end{array}$ & $\begin{array}{c}\text { No (\%) } \\
\text { confirmed }\end{array}$ & & $\begin{array}{c}\text { No } \\
\text { tested }\end{array}$ & $\begin{array}{c}\text { No }(\%) \\
\text { confirmed }\end{array}$ \\
\hline$<1$ & 36 & $4(11)$ & & & \\
$1-4$ & 25 & $5(20)$ & & $43^{\star}$ & \\
$5-9$ & 13 & $6(46)$ & & 28 & $3(11)$ \\
$10-14$ & 44 & $37(84)$ & & 11 & $7(64)$ \\
$15-24$ & 22 & $17(77)$ & & 5 & $3(60)$ \\
$\geqslant 25$ & 9 & $1(11)$ & & & \\
\hline Total & 149 & $70(47)$ & & 87 & $13(15)$
\end{tabular}

*Includes two children aged 14 months positive for IgM antibody who received measles, mumps, and rubella vaccine 4-6 weeks before sample collection in whom measles specific IgM was presumed to be vaccine induced.

TABLE II-Detection rate for measles specific IgM in sporadic and outbreak associated notified cases of measles

\begin{tabular}{lccccc}
\hline & \multicolumn{2}{c}{ Sporadic cases } & & \multicolumn{2}{c}{ Outbreak associated cases } \\
\cline { 2 - 3 } \cline { 6 - 7 } $\begin{array}{l}\text { Age } \\
\text { (years) }\end{array}$ & $\begin{array}{c}\text { No } \\
\text { tested }\end{array}$ & $\begin{array}{c}\text { No }(\%) \\
\text { confirmed }\end{array}$ & & $\begin{array}{c}\text { No } \\
\text { tested }\end{array}$ & $\begin{array}{c}\text { No }(\%) \\
\text { confirmed }\end{array}$ \\
\hline$<1$ & 34 & $2(6)$ & & 2 & $2(100)$ \\
$1-9$ & 100 & $7^{\star}(7)$ & & 9 & $7(78)$ \\
$10-14$ & 18 & $12(67)$ & & 37 & $32(86)$ \\
$\geqslant 25$ & 20 & $8(40)$ & & 16 & $13(81)$ \\
\hline Total & 172 & $29(17)$ & 64 & $54(84)$
\end{tabular}

ॠxcludes two cases in which measles specific IgM was presumed to be vaccine induced.

TABLE II-Detection of measles specific IgM in paired serum and saliva specimens collected from 236 patients notified as having measles

Saliva IgM

Positive Negative Total

\begin{tabular}{lrrr}
\hline Serum IgM: & & & \\
Positive & 71 & 14 & 85 \\
Negative & 3 & 148 & 151 \\
\hline Total & 74 & 162 & 236 \\
\hline
\end{tabular}

weekly intervals; both approaches gave similar results. Vaccine efficacy was estimated by using the screening method with age stratification' and national figures for vaccine coverage in the annual birth cohorts eligible for measles or measles, mumps, and rubella vaccination. $^{2} 10$

\section{Results}

Paired serum and saliva samples taken within 90 days of onset of illness were obtained for 236 measles cases notified between August 1991 and February 1993. Measles specific IgM was detected in 85 (36\%) serum samples. The detection rate declined with increasing time between onset and sampling $(P<0.001$ for the linear trend). For instance, for samples taken within six weeks of onset $80 / 194(41 \%)$ were positive compared with $5 / 42(12 \%)$ for those taken seven to 12 weeks after onset. A documented history of measles vaccine or measles, mumps, and rubella vaccine was obtained for 87 (37\%) patients, 10 of whom had received both vaccines. In every age group, proportionately fewer vaccinated than unvaccinated patients were positive for measles specific IgM (table I). The proportion of confirmed cases in those who were vaccinated, $13 / 86(16 \%)$, was consistent with a vaccine efficacy of $88 \%$ (95\% confidence interval 70 to $95 \%$, age stratified). Excluding the two recently vaccinated patients in whom measles specific $\operatorname{IgM}$ antibody was presumed to be induced by vaccine, the median interval between vaccination and infection was 11 years (range 1.5 to 14 ). Only one of the 13 vaccinated patients with confirmed infection had been vaccinated before 15 months of age; none had received two doses of vaccine.

Two districts experienced outbreaks centred on local secondary schools during the study period; 54/64 $(84 \%)$ of outbreak associated cases were positive for measles specific IgM compared with $29 / 172$ (17\%) of sporadic cases $\left(\mathrm{P}<0.0001, \chi^{2}\right.$ test) (table II). Among outbreak associated cases investigated within 42 days of onset, 53/57 (93\%) had specific IgM in serum. Age, vaccination status, and case type were independently associated with detection of measles $\operatorname{IgM}(\mathrm{P}<0.001$ for each factor, tables I and II). Three of the four outbreak associated cases negative for measles specific IgM in serum were in unvaccinated secondary school children with typical measles, two of whom had measles specific IgM antibody in saliva. The fourth was in a 5 year old child who had received measles, mumps, and rubella vaccine three years previously and was admitted to hospital with clinical diagnosis of severe measles; paired serum samples taken one day and six weeks after onset of rash showed a static measles titre (64) by haemagglutination inhibition.

Table III shows the concordance of detection of measles IgM in serum and saliva. Overall, there was agreement on $219 / 236$ (93\%) paired serum and saliva samples. Measles specific IgM was detected in saliva of $71 / 85(84 \%)$ patients with measles specific IgM in serum. Of the 14 patients with positive serum and negative saliva, five had no detectable total IgG antibody in saliva, suggesting that an inadequate specimen had been collected, and in three of the remaining nine patients the interval between onset and sample collection was over 42 days; of the specimens from patients concordant, and positive, for measles specific IgM, only $2 / 71$ had had samples collected after 42 days. Thus, IgM was detected in $71 / 77$ (92\%) adequate saliva samples taken from patients positive for serum IgM within 42 days of onset of measles. In three outbreak associated cases with typical measles, IgM was detected in saliva but not in serum IgM (two described above).

Serum samples from notified cases in which measles IgM was not detected were tested for the presence of IgM specific for rubella and human parvovirus B19. Rubella specific IgM was detected in $6 / 101$ and parvovirus $B 19$ in 5/132 samples investigated.

\section{Discussion}

Since 1940 national surveillance of measles in England and Wales has relied on the statutory notification system. This is based on the reporting doctor's clinical diagnosis, and no laboratory confirmation is required. Our study shows that this surveillance system lacks the precision required for effective measles control. The low rate of serological confirmation of notified cases is unlikely to be due to inadequacy of the antibody capture radioimmunoassay technique since specific IgM was detected in the serum of $93 \%$ of outbreak associated cases investigated within six weeks of onset. Rubella and parvovirus B19 seem to be responsible for a minority of incorrectly diagnosed measles cases in the United Kingdom, and other infectious causes of measles-like illness need to be sought.

Our findings have implications for vaccination policy. For example, the recent increase in the proportion of notified cases in chidlren under a year old may be spurious as infection was confirmed in only $11 \%$ of cases in this age group. This suggests that infants do not have a major role in maintaining transmission and that reduction in the age at immunisation is therefore not indicated at present. On the other hand, notifications probably underestimate the true proportion of measles cases occurring in older age groups. Secondary school outbreaks and age specific immunity to measles are being investigated further to assess whether supplementary vaccination of older children is required. Also, without confirmation of clinical diagnoses, the role of vaccine failure in sustaining transmission is likely to be overestimated and a proper investigation of the risk factors for vaccine failure cannot be undertaken. The proportion of confirmed cases in vaccinated patients $(16 \%)$ was consistent with a vaccine efficacy of around $90 \%$, similar to that found for live monovalent measles vaccine in the Medical Research Council trial. ${ }^{11}$ Results to date do not suggest that vaccine failure is 


\section{Public health implications}

- Disease surveillance is the cornerstone of programmes for measles control

- Surveillance based on clinically diagnosed measles cases is innaccurate-a clinical diagnosis of measles is confirmable by laboratory tests in only about one third of notified cases

- Of notified cases with a documented history of vaccination, $15 \%$ are confirmed as measles

- This study found agreement in detecting measles in $93 \%$ of paired serum and saliva samples; agreement was more likely if an adequate amount of saliva had been collected within 42 days of the onset of measles

- Salivary testing is a suitable non-invasive method for confirming clinical diagnosis of measles and could have an important role in controlling the disease

associated with immunisation before 15 months of age, a factor that has been identified in American studies. ${ }^{12}$

Clinical measles, confirmed by a significant rise in measles antibody, but without an IgM response, has been described in previously vaccinated individuals. ${ }^{13}$ Our study suggests that this is uncommon, as all but one of the outbreak associated cases in vaccinated patients investigated within six weeks of onset were positive for IgM, and measles infection in the remaining patient was excluded on serological grounds. However, the use of alternative methods of confirming infection by virus isolation and virus detection by polymerase chain reaction is planned in order to assess the frequency of IgM negative infections and to investigate the role of subclinical cases in transmission.

There was good overall agreement between serum and saliva specimens in the detection of measles specific IgM, with $93 \%$ of paired samples giving concordant results. However, IgM was detected in saliva in only $84 \%$ of cases with positive results in serum. One factor which contributed to a missed salivary diagnosis was late collection of the sample. We previously showed that measles specific IgM was detectable in saliva for six weeks after the onset of infection. ${ }^{5}$ The present study indicates that its detection after this time is unreliable. A second factor contributing to missed salivary diagnoses was inadequate sample collection. Improved saliva collection devices are being evaluated, but for the present the adequacy of the saliva sample should be confirmed to avoid false negative results. The three cases with positive results in saliva and negative results in serum may represent a low rate of false positive salivary results. However, their exclusive occurrence in clinically typical cases associated with outbreaks suggests that the salivary diagnosis was correct in these instances. In saliva samples containing immunoglobulin and collected within six weeks of onset of measles,
IgM was detected in $92 \%$ of cases with serological confirmation. This level of sensitivity would make salivary IgM detection a suitable non-invasive method for routine investigation of notified cases.

Accurate surveillance is essential for effective strategies for eliminating measles. Surveillance programmes in the Americas use a strict clinical case definition and recommend serological confirmation or epidemiological linkage of clinical cases before control measures are instituted. ${ }^{14}$ However, the requirement to take blood samples inevitably limits the scale of laboratory investigation, and expensive and unnecessary control measures have been instituted on the basis of false positive clinical diagnoses. ${ }^{15}$ Our study indicates that detection of measles IgM in saliva is an effective alternative to detection in serum and suggests that salivary diagnosis could have a major role in measles control programmes. Work aimed at producing a simpler assay that would enable widespread saliva testing is under way.

We thank the consultants in communicable disease control and general practitioners in the following districts for their help: Barnet; Bedfordshire; Dorset; East and North Hertfordshire, East, North, South and West Birmingham; Eastern Health Board (Ireland); East Sussex; Gloucester; Isle of Man; Mid Downs; Mid Essex; Mid Surrey; North East Essex; North Yorkshire; South West Durham; St Helens and Knowsley; Merton, Sutton and Wandsworth; South East Kent; West Cumbria. We also thank Mrs S Cole and Mrs J Vurdien for administrative assistance, Mrs $P$ Kaye for help with data analysis, Dr P Farrington for statistical advice, Dr B Cohen for conducting the parvovirus B19 assays, and the Department of Health for providing financial support for the laboratory work.

1 Department of Health. Immunisation against infectious disease. London: HMSO, 1988.

2 White JM, Leon S, Begg NT. COVER (Cover of vaccination evaluated rapidly):25. Communicable Diseases Report 1993;3:R71-2.

3 Miller E, Nokes DJ, Anderson RM. Measles, mumps, and rubella vaccination. BMF 1992;304:1440-1.

4 Carter H, Gorman D. Measles, mumps and rubella vaccine: time for a two stage policy. $B M \Im$ 1992;304:637.

5 Perry KR, Brown DWG, Parry JV, Panday S, Pipkin C, Richards A. The detection of measles, mumps and rubella antibodies in saliva using antibody capture radioimmunoassay. $¥$ Med Virol 1993;40:235-40.

6 Mortimer PP, Parry JV. Non-invasive virological diagnosis: are saliva and Mortimer PP, Parry JV. Non-invasive virological diagnosis: are saliva and
urine specimens adequate for blood? Reviews in Medical Virology 1991;1: 73-8.

7 Faraclegan H, Quinn T, Polk BF. Detecting antibodies to human immunodeficiency virus in dried blood on filter papers. $f$ Infect Dis 1987;155:1073-4.

8 Cohen BJ, Mortimer PP, Pereira MS. Diagnostic assays with monoclonal antibodies for the human serum parvovirus-like virus (SPLV). $f$ Hyg (Camb) 1985;91:113-30.

9 Farrington CP. Estimation of vaccine effectiveness using the screening method. Int f Epidemiol 1993;22:742-6.

10 Department of Health, Statistics Division. Form SBL607, 1966-86; Form KC51 1987;1990/91. London: DoH, 1966-91.

11 Fourth report to the Medical Research Council by the Measles Sub-Committee on Development of Vaccines and Immunisation Procedures. Lancet 1977;ii:571-5.

12 Hull HF, Montes JM, Hays PC, Lucero RL. Risk factors for measles vaccine failure among immunized students. Pediatrics 1985;76:518-23.

13 Reyes MA, de Borrero MF, Roa J, Bergonzoli G, Saravia NG. Measles vaccine failure after documented seroconversion. Pediatr Infect Dis $f$ 1987;6:848-51.

14 Hinman AR, Kirby CD, Eddins DL, Orenstein WA, Bernier RH, Turner PN, et al. Elimination of indigenous measies from the United States. Rev Infect Dis 1983;5:538-45.

15 Robertson SE, Markowitz LE, Berry DA, Diny EF, Orenstein WA. A million dollar measles outbreak: epidemiology, risk factors and a selective revaccination strategy. Public Health Rep 1992;107:24-31.

(Accepted 11 fanuary 1994) 